\title{
Multiple Sclerosis: Effect of Oral Administration of an Antioxidant Dietary Supplement in C57BL6/N Induced Model of Experimental Autoimmune Encephalomyelitis
}

\author{
Torricelli Piera ${ }^{1}$, Antonelli Francesco ${ }^{2}$, Ferorelli Pasquale ${ }^{2}$, De Martino Angelo², \\ Shevchenko Anna ${ }^{3}$, Beninati Simone ${ }^{2}$ \\ ${ }^{1}$ Department SPES, University of Molise, Campobasso, Italy \\ ${ }^{2}$ University of Tor Vergata, Department of Biology, Rome, Italy \\ ${ }^{3}$ People's Friendship University of Russia, Department of medicine, Moscow, Russia
}

Email address:

beninati@bio.uniroma2.it (Beninati S.)

\section{To cite this article:}

Torricelli Piera, Antonelli Francesco, Ferorelli Pasquale, De Martino Angelo, Shevchenko Anna, Beninati Simone. Multiple Sclerosis: Effect of Oral Administration of an Antioxidant Dietary Supplement in C57BL6/N Induced Model of Experimental Autoimmune Encephalomyelitis. American Journal of Clinical and Experimental Medicine. Vol. 3, No. 2, 2015, pp. 83-87. doi: 10.11648/j.ajcem.20150303.12

\begin{abstract}
In the pathogenesis of demyelinating diseases including multiple sclerosis (MS) an important role is played by oxidative stress. Increased energy requirements during remyelination of axons and mitochondria failure is one of the causes of axonal degeneration and disability in MS. In the presence of neurological diseases such as MS, F2-isoprostanes are concentrated in higher quantities. In this context, we analyzed the levels of F2-isoprostanes in the plasma and cerebrospinal fluid of mice of the strain C57BL6/N with an induced experimental autoimmune encephalomyelitis (EAE), orally treated with two concentrations of Citozym, a dietary supplement with evident antioxidant properties. Compared to the control group, Citozym-treated EAE-mouse had significantly lower levels of F2-isoprostanes both in plasma and in cerebrospinal fluid. Furthermore, according to the guidelines of IACUC, treatment with Citozym at higher concentrations drastically reduced neurological signs of induced EAE.
\end{abstract}

Keywords: Food Supplements, Antioxidants, Multiple Sclerosis, F2-Isoprostanes, Oxidative Stress

\section{Introduction}

MS is a disease that affects the white matter of the central nervous system (CNS), brain and spinal cord. It is a demyelinating disease. Inflammatory demyelinating lesions are localized in the myelin sheaths that as sleeves are of the nerves. Myelin well has a protective role, allows the nervous impulse to travel quickly along the nerve, thus ensuring a perfect nervous stimulus conduction. In this disease the loss of myelin (demyelination) leads to a slowing of nerve impulse and therefore to the appearance of numerous neurological symptoms. It has long been known that reactive oxygen species (ROS) and nitrogen (RNS) can have a double meaning in the human malignant or benign pathophysiology. The overproduction of ROS / RNS cause oxidative or nitrosative stress, which follows the alteration of cellular structures, including membranes, lipids, proteins and DNA. In contrast, at low concentrations of ROS / RNS corresponds to a physiological role in the regulation of cellular responses, first by a modulation of signal transduction pathways. It is also not resolved the question of whether the formation of ROS / RNS is always a primary cause or, sometimes, just a result of tissue damage. The CNS is particularly vulnerable to oxidative processes due to the high amount of oxygen required by the neuronal metabolism, the high content of unsaturated fatty acids, easily attacked by the peroxidation, in the phospholipids of cell membranes and myelin, and the presence, especially in certain regions of the brain, of significant deposits of redox-active metals such as iron or copper. The results indicate that the deficit of lipophilic antioxidants in blood of MS patients may have a negative impact on bioenergetics of reparative remyelinating processes and promote neurodegeneration. An energy deficient state and oxidative and nitrosative stress have been implicated in the degeneration of axons in MS [1]. In chronic lesions, axonal degeneration correlates with the extent of inflammation and leads to axonal loss through a slow burning process [2]. A 
lipid peroxidation disturbance, caused by free radical production is probably involved in the breakdown of the myelin sheath [3]. Since then several studies have demonstrated the role of increased free radical production and/or a decreased antioxidant defense in CNS as causal factors of MS [4]. Following demyelination the axonal membrane undergoes a number of changes including an increase in number of sodium channels within demyelinated part of the axon [5]. The maintenance of intra-axonal ionic balance and resting membrane potential following the influx of sodium through the increased sodium channels relies on the largest consumer energy in the CNS, [6]. In non inflammatory environments, this increase in energy demand of axons lacking a healthy myelin sheath is apparent by the changes in density and activity of energy producing organelles mitochondria, the most efficient producers of energy [7]. Though the principal site of MS pathology is the CNS, the lipid status and the membrane properties in the platelets and erythrocytes in the peripheral blood are also altered [8]. Increased lipid peroxide levels have been observe both in the cerebrospinal fluid (CSF) and in the blood of MS patients [9]. Lack of sufficient vitamin $\mathrm{A}$ and $\mathrm{E}$ in the diet has been suggested to be a risk factor for the onset of the disease. The F2-isoprostanes are markers that identify oxidative stress. They are part of the family of eicosanoids, local hormones derived from the metabolism of polyunsaturated fatty acids of the Omega-3 family and Omega-6. F2-isoprostanes are a unique prostaglandin-like products, which are formed via nonenzymatic, free radical-mediated peroxidation of polyunsaturated fatty acids. Since the production of an eicosanoid influence on the action and on the production of almost all other hormones, F2-isoprostanes have essential functions for the regulation of the activities and cellular functions [10]. In the presence of neurological diseases such as MS, they are concentrated in the plasma and CSF in higher quantities. Especially in relapsing remitting MS, the levels observed increase as the disease progresses. In conclusion, although still not been clarified how it is caused in MS, neuro-axonal damage, it is well established the substantial role of oxidative mechanisms. Induction of EAE in rodents is a widely used approach for investigation of the pathophysiological mechanisms involved in the MS clinical disease course and for the evaluation of potential novel therapeutics for disease management [11]. Our objective was to understand the role of oxidative stress on the neurological signs, a pathological hallmark of MS, in modulating the plasma and CSF levels of F2-isoprostanes as markers, by a dietary supplement with antioxidant properties called Citozym (CIZ).

\section{Experimental Protocol}

\subsection{Animal Treatments}

Male C57BL6/N mice were purchased from IFFA Credo (L'Abreole, France). Mice were housed throughout the experiments in air-conditioned animal room and fed with a
AIN-76 purified rodent diet composition (Dyets, Inc. Bethlehem, PA USA). All experimental protocols were carried out following the Guidelines for the Welfare of Animals in Experimental Research and the ECC Council Directive 86=609, OJL 358, 1st December 1987. 50 mice were treated for induction of EAE [12].

Three groups of animals were used for this investigation and observed according to the IACUC guidelines (http://www.upenn.edu/regulatoryaffairs/Documents/iacuc guideline-eaedemyelinatingrodentmodels.pdf). Each group was reared in separate cages and checked daily. Group A: 10 control animals with induction of EAE, orally treated with sucrose diluted in water to $50 \%$ twice a day for 63 days. Group B: 20 animals with induction of EAE orally treated twice a day for 63 days with CIZ diluted in water to $25 \%$ (1: $4 \mathrm{v} / \mathrm{v})$. Group C: 20 animals orally treated with induction of EAE treated twice a day for 63 days with CIZ diluted in water to $50 \%$ (1:2 $\mathrm{v} / \mathrm{v}$ ). CIZ was obtained from CITOZEATEC, S.r.l. (Peschiera Borromeo,Milano, Italy)). The main components of CIZ are as follows (units/100 g): $500 \mathrm{mg}$ of vitamin C, $56 \mathrm{mg}$ of vitamin B5, $56 \mu \mathrm{g}$ of vitamin D, 3,3 $\mathrm{mg}$ of vitamin B9, 222 $\mathrm{mg}$ of pyruvic acid, $120 \mathrm{mg}$ of citric acid, $250 \mathrm{mg}$ of tartaric acid and $77.8 \mathrm{~g}$ of carbohydrates.

\subsection{Observation and Evaluation of Clinical Signs}

Animals were observed daily for the control of clinical signs of EAE and classified according to the IACUC guides (Table I). The data are reported as the observation of a number of animals greater than $75 \%$ of the total.

Table I. EAE clinical signs and IACUC score.

\begin{tabular}{ll}
\hline Score/stage & EAE Clinical Signs \\
\hline 0 & Clinically Normal \\
1 & Weak Tail \\
2 & Weak hind limbs \\
3 & Paralyzed hind limbs \\
4 & Weak front limbs \\
& Paralyzed hind limbs \\
5 & Paralyzed front/hind Limbs \\
6 & Moribond \\
\hline
\end{tabular}

\subsection{Bioassays}

Evaluation of the levels of F2-isoprostanes in the blood plasma and CSF of animals was carried out through the use of techniques GC / MS and LC / ESI / MS / MS [13].

\subsection{Biological Sampling}

The blood sampling was performed every 7 days from the outer ear of the animal. From this it has been prepared the plasma. The CSF was collected every 7 days for lumbar puncture. The three sets of samples (A, B and C) were analyzed for levels of F2-isoprostanes indicated in the experimental protocol. 


\subsection{Statistical Analyses}

The statistical analysis was performed using the Mann-Whitney $\mathrm{U}$ test for intergroup comparisons. Spearman's rank correlation test and the linear regression analysis were performed to analyze correlations between groups $\mathrm{A} / \mathrm{B}$ and $\mathrm{A} / \mathrm{C}$ and the duration of administration of $\mathrm{CIZ}$ and to draw least squares regression lines. Values were expressed as the mean \pm SD. $P<0.05$ was regarded as statistically significant

\section{Results}

Treatment with CIZ at higher concentrations has drastically reduced neurological signs of induced EAE (Table II and Figure 1). The control mice (group A) showed clear signs of quadriplegia after 42-49 days from induction of EAE. After 56-63 days showed signs of respiratory distress and in accordance with the guidelines of IACUC were sacrificed. CIZ-treated animals (1: $4 \mathrm{v} / \mathrm{v}$, group B), showed paraplegia and urinary incontinence after 28-35 days of treatment, getting worse and reaching the status of quadriplegia towards the end of treatment (56-63 days). CIZ-treated animals (1:2 v/v group C) instead have maintained the condition of paraplegia and urinary incontinence without worsening, until the conclusion of the experiment. In particular, group $\mathrm{C}$ shows an evident slowing down of symptoms compared to the control group A and group B. Since EAE is a demyelinating disease very similar to SM, these results could be extended to SM in the humans.

Table II. neurological signs of induced EAE estimated by IACUC score.

\begin{tabular}{llll}
\hline Group & A & B & C \\
\hline Days & EAE & Clinical & Signs \\
$0-7$ & 0 & 0 & 0 \\
$7-14$ & 1 & 1 & 1 \\
$14-21$ & 3 & 2 & 1 \\
$21-28$ & 3 & 2 & 2 \\
$28-35$ & 4 & 3 & 3 \\
$35-42$ & 4 & 3 & 3 \\
$42-49$ & 5 & 4 & 3 \\
$49-56$ & $>5$ & 4 & 3 \\
$56-63$ & 6 & 5 & 3 \\
\hline
\end{tabular}

The plasma and the CSF levels of F2-isoprostanes were reduced following treatment with $\mathrm{CIZ}$ at the two concentrations used. The cascade of reactions leading to the formation of F2-isoprostanes, but also of other chemical species, takes the name of "lipid peroxidation" and thus allows to evaluate the antioxidant power of a supplement or a drug. The levels and the identification of in vivo neuroprostanes was carried out through the use of techniques GC / MS and LC / ESI / MS / MS [13]. This latter technique proved to be especially important for the characterization of the various series of neuroprostanes and their quantification. Since the neuroprostanes are esterified to phospholipids, the sample was hydrolyzed with chemical methods ( $\mathrm{KOH}$ or phospholipase). The formation of neuroprostanes, in analogy with what happens for the F2-isoprostanes or prostaglandins, occurs by oxidation of unsaturated fatty acids in the cell membranes. The F2-isoprostanes Series 2 and prostaglandins derived from the oxidation of arachidonic acid, the F3-isoprostanes Series 3 from eicosapentaenoic acid (EPA) and the precursor of neuroprostani is docosahexaenoic acid (DHA). In recent years the determination of levels of F2-isoprostanes in tissues and biological fluids has significantly improved our knowledge of the role played by oxidative stress in many neurological diseases [10].

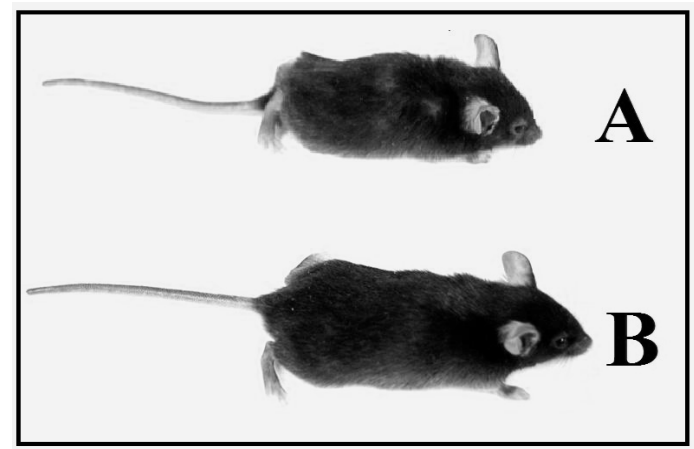

Figure 1. Representative mice of the strain C57BL6/N A) untreated and B) treated with CIZ 1:2 v/v for 63 days.

Increased levels of F2-isoprostanes indicates a strong lipid peroxidation, while the reduction is a sign of antioxidant action. To this end we have assessed the levels of these molecules, in order to correlate the variation of neurological signs following treatment with CIZ, in groups B and C, to the antioxidant potentially exerted by this food supplement. There is a clear reduction in the levels of F2-isoprostanes in both plasma and CSF of the animals treated with CIZ at the two concentrations used (Figure 2 and 3 ).

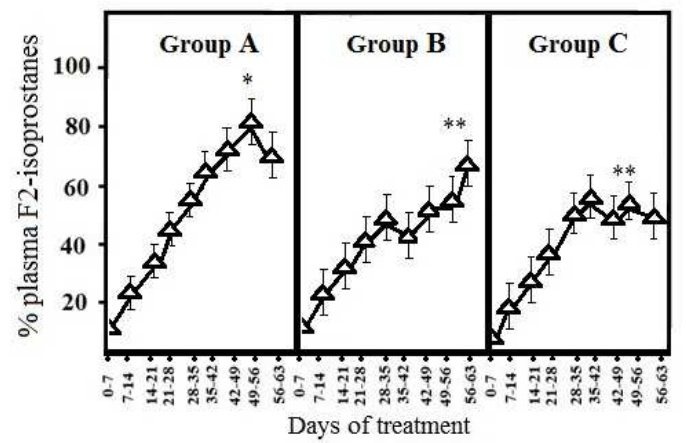

Figure 2. Plasma levels of F2-isoprostanes in the three groups of mice of the strain C57BL6/N. The value is the average of four determinations \pm S.D. Statistically significant $(P<0.05)$ reduction of $F 2$ isoprostanes levels $(* *)$ is reported in comparison to control $(*)(\mathrm{n}=15)$

\section{Discussion}

MS is one of the most common diseases that affect the CNS. It is an inflammatory, demyelinating, chronic and often progressively disabling disease [14]. In MS, it is thought that an autoimmune reaction abnormal assaults and destroys the myelin (demyelination), resulting in open areas of healed skin, called plaques or lesions, along the nerve. This means that the 
immune system reacts against a component of the body as a foreign material, attacking and destroying it as it would with any external aggressor. The conduction of nerve impulses is consequently slowed or blocked. For these reasons, EAE is an excellent model of MS [11]. The causes of MS are not yet known. Based on the results of many scientific studies are believed to be involved more factors that interact: a genetic predisposition and environmental factors that can stimulate the immune system. The external factors, environmental, would be crucial both for the induction of the disease which for its maintenance with the unleashing of relapses. Even vaccinations may precede the onset of the disease is that the unleashing of relapses [15]. Many viruses and some bacteria were repeatedly indicted as responsible for the disease, but none of these has ever been proven. Probably it is not important to a single specific agent, but may be the same process of infection, especially when this takes place in a certain moment of time corresponding to a particular state of immunity of the patient. MS is a disease spread worldwide. In Italy 54,000 people are affected by MS, one every 1,100 inhabitants. Every year there are 1,800 new cases. The age of onset is between 15 and 50 years, although this disease occurs mainly among young adults, between 20 and 30 years, and among women, in ratio of 2 to 1 compared to men [16].

The symptoms of MS are varied. Their variety is because the demyelinating lesions (plaques) are typical of the disease can affect different areas of the CNS (brain, spinal cord and optic nerve). Depending on its location, a plaque can cause such as a motor disorder in a lower limb, a vision problem or a tingling in the hand and arm. Most patients have an abrupt onset with subsequent relapses and remissions. Only a minority has a rapid progressive clinical deterioration. The deterioration is usually slow and occurs with no relapses followed by a complete functional recovery; here as there is a growing permanent disability. The treatment of MS involves forms of intervention in relation to the stage of the disease, the type of course and severity of the clinical picture [16].

Much current MS research is focused on trying to clarify the exact nature of the autoimmune response against myelin, in order to counter it, and on trying to find the factors that allow remyelination of damaged nerves. It was shown that remyelination occurs spontaneously in some lesions and studies on animal model of MS have demonstrated that some drug compounds are able to induce remyelination in demyelinated nerves.

Recently, it has been characterized another class of products derived from lipid peroxidation, isoprostanes, which have several significant advantages over the indicators of oxidative stress classically used [17]. These benefits are mainly associated to their stability, relative abundance and availability of specific and sensitive analytical methods to measure them in body tissues and fluids. The CNS is particularly vulnerable to oxidative processes due to the high amount of oxygen required by the neuronal metabolism, the high content of unsaturated fatty acids, easily attacked by the peroxidation, in the phospholipids of cell membranes and myelin and the presence, especially in certain regions of the brain, of significant deposits of redox-active metals such as iron or copper. The oxidation-reduction thus triggered (redox cycling) precisely lead to the formation of ROS, such as the hydroxyl radical is particularly active in the initiation of lipid peroxidation. Similar phenomena of redox cycling seem to participate in the pathogenesis of hitting in MS [18]. Moreover, complex interactions, in degenerative processes, involve also glutathione, the NO and dopamine, which have the same ability to catalyze reactions of redox cycling of iron and copper. We report the use of a mixture of components with a high antioxidant power [19], in animals in which EAE was induced, that allowed to control, the potential usefulness in reducing neurological symptoms, estimated following the IACUC international guidelines and to evaluate a biochemical marker of oxidative stress [13], the F2-isoprostanes, which currently has proven useful in quantifying the extent of neurological damage [17]. The results of the experiments presented in this report, clearly show a slowing, in animals suffering from EAE, of neurological damage, following the oral administration of CIZ ( $50 \%$ in water $\mathrm{v} / \mathrm{v})$ twice a day for 63 days. The assessment of the levels of F2-isoprostanes performed in both plasma and CSF, has highlighted and confirmed [19-21], the powerful antioxidant action of CIZ, which led to a marked reduction in the concentration of these molecules. In conclusion we can affirm that the reported positive effects of CIZ, could be the basis for a potential trial on MS patients with the intention of improving the quality of life of these subjects.

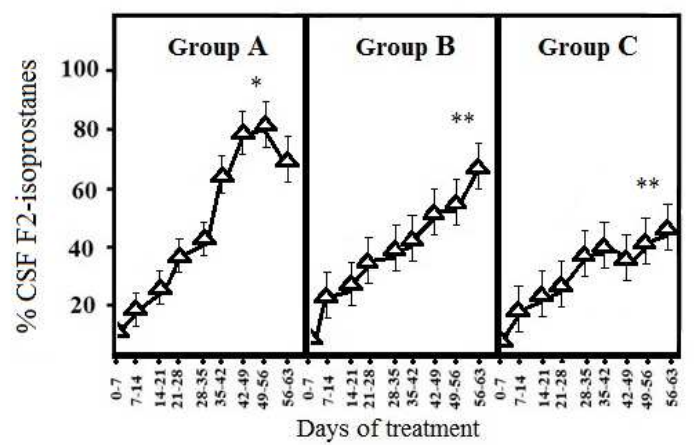

Figure 3. CSF levels of F2-isoprostanes in the three groups of mice of the strain C57BL6/N. The value is the average of four determinations $\pm S . D$. Statistically significant $(\mathrm{P}<0.05)$ reduction of $F 2$ isoprostanes levels $\quad\left({ }^{* *}\right)$ is reported in comparison to control (*) $(\mathrm{n}=15)$

\section{Acknowledgements}

We are grateful to A.I.A.S Italy, for financial support for studying the possibility of alternative therapies for Multiple Sclerosis.

\section{References}

[1] B. Uttara, A. V. Singh, P. Zamboni, and R. T. Mahajan, "Oxidative stress and neurodegenerative diseases: a review of upstream and downstream antioxidant therapeutic options. Current Neuropharmacology, 2009; 7(1): 65-74 
[2] R. Dutta and B. D. Trapp, "Pathogenesis of axonal and neuronal damage in multiple sclerosis. Neurology. 2007;68 (22): S22-S31.

[3] H. S. Mickel, "Multiple sclerosis: a newhypothesis. Perspectives in Biology and Medicine. 1975; 18 (3): 363-374.

[4] H. T. Besler and S. Comoglu. Lipoprotein oxidation, plasma total antioxidant capacity and homocysteine level in patients with multiple sclerosis. Nutritional Neuroscience. 2003; 6 (3):189-196.

[5] J. A. Black, J. Newcombe, B. D. Trapp, and S. G. Waxman, Sodium channel expression within chronic multiple sclerosis Plaques. Journal of Neuropathology and Experimental Neurology. 2007; 66 (9): 828-837.

[6] B. D. Trapp, and S. G. Waxman, Sodium channel expression within chronic multiple sclerosis Plaques. Journal of Neuropathology and Experimental Neurology. 2007; 66 (9): 828-837.

[7] A. Ames III. Central nervous system energy metabolism as related to function. Brain Research Reviews. 2000; 34 (1-2): $42-68$.

[8] H. Andrews, K. White, C. Thomson et al. Increased axonal mitochondrial activity as an adaptation to myelin deficiency in the shiverer mouse. Journal of Neuroscience Research. 2006; 83 (8): 1533-1539.

[9] E. Karg, P. Kliv'enyi, I. N'emeth, K. Bencsik, S. Pint'er, and L. V'ecsei. Nonenzymatic antioxidants of blood in multiple sclerosis. Journal of Neurology. 1999; 246 (7):533-539.

[10] GL. Milne, Q. Dai, LJ. Roberts LJ 2nd. The isoprostanes-25 years later. Biochim Biophys Acta. 2015; 1851(4):433-445.

[11] R.Gold, C. Linington, H. Lassmann. Understanding pathogenesis and therapy of multiple sclerosis via animal models: 70 years of merits and culprits in experimental autoimmune encephalomyelitis research. Brain. $2006(\mathrm{Pt}$ 8):1953-1971.

[12] K. O'Brien, D. Fitzgerald, A.Rostami, B.Gran. The TLR7 agonist, imiquimod, increases IFN-beta production and reduces the severity of experimental autoimmune encephalomyelitis. J Neuroimmunol. 2010;221(1-2):107-111
[13] GL. Milne, H. Yin, JD. Brooks, S. Sanchez, L. Jackson Roberts 2nd, JD. Morrow JD. Quantification of F2-isoprostanes in biological fluids and tissues as a measure of oxidant stress. Methods Enzymol. 2007;433:113-126.

[14] DH. Mahad, BD. Trapp, H. Lassmann H. Pathological mechanisms in progressive multiple sclerosis. Lancet Neurol. 2015;14(2):183-193

[15] A. Langer-Gould, L. Qian, SY. Tartof, SM. Brara, SJ. Jacobsen, BE. Beaber, LS. Sy, C. Chao, R. Hechter, HF. Tseng. Vaccines and the risk of multiple sclerosis and other central nervous system demyelinating diseases. JAMA Neurol. 2014; 71(12):1506-1513

[16] D. Ontaneda, RJ. Fox, J. Chataway. Clinical trials in progressive multiple sclerosis: lessons learned and future perspectives. Lancet Neurol. 2015; 14(2):208-223.

[17] VK. Harris, SA. Sadiq. Biomarkers of therapeutic response in multiple sclerosis: current status. Mol Diagn Ther. 2014; 18(6):605-617.

[18] G.G. Ortiz, F. P. Pacheco-Moisés, O. K. Bitzer-Quintero, A. C. Ramírez-Anguiano, L. J. Flores-Alvarado, V. Ramírez -Ramírez, M. A. Macias-Islas, E. D. Torres-Sánchez. Immunology and Oxidative Stress in Multiple Sclerosis. Clinical and Basic Approach. Neuroscience Letters. 2007; 414(3):233-236.

[19] P. Torricelli, P. Ferorelli, A. De Martino, F. Antonelli, A. Shevchenko, S. Beninati. Regression of Carotid Plaques in Individuals at Low-to-intermediate Cardiovascular Risk Treated with Citozym and Propulzym. European Journal of Preventive Medicine. 2014; 2 (3): 33-37.

[20] P. Torricelli, P. Ferorelli, A. De Martino, F. Antonelli, S.Beninati. Preventive Effects of A Mixture of Micronutrients with antioxidative Properties on Experimentally Induced Prostate Hyperplasia. American J Life Sci. 2013; 1(1): 22-26.

[21] P. Torricelli, F. Antonelli , P. Ferorelli, A. De Martino , A. Shevchenko , S. Beninati. Antiproliferative Activity of a Dietary supplement on Estrogen Receptor Positive and Negative Human Breast Adenocarcinoma Cell Lines. Cancer Research Journal. 2014; 2 (2), 2014:29-32. 